\title{
Innovación basada en metodologías activas para el aprendizaje en la asignatura Pirometalurgia de la Universidad Técnica Federico Santa María, Chile
}

\author{
Innovation based on active methodologies for learning in the pyrometallurgy
} course at the Technical University Federico Santa María, Chile

Inovação baseada em metodologias ativas para o aprendizado no curso de Pirometalurgia da Universidade Técnica Federico Santa María, Chile

Héctor Henao

Universidad Técnica Federico Santa María, Chile hector.henao@usm.cl https://orcid.org/0000-0002-4212-5982

Alejandra Chávez Universidad Técnica Federico Santa María, Chile alejandra.chavez@usm.cl https://orcid.org/0000-0002-5086-2683

Claudio Pizarro CODELCOTECH, Chile Claudio.e.pizarro.r@gmail.com https://orcid.org/0000-0002-5605-4310

Christian García Universidad Técnica Federico Santa María, Chile christiangarciaordenes@gmail.com https://orcid.org/0000-0002-7571-509X

Juan Ibáñez Universidad Técnica Federico Santa María, Chile 


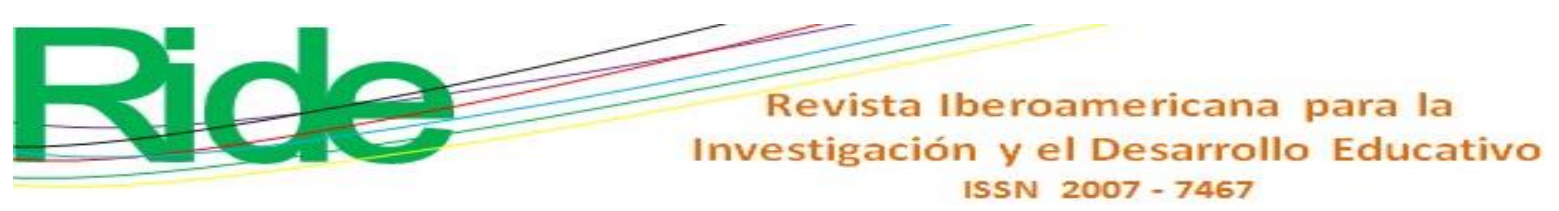

efectivamente a la participación de los estudiantes en clase, promovieron el dialogo en actividades grupales y crearon mejores condiciones de aprendizaje.

Palabras clave: innovación metodológica, metodología activa de aprendizaje, modelo constructivista, Pirometalurgia, rediseño de curso.

\section{Abstract}

This paper describes an innovation in the teaching methodology of the pyrometallurgy course, belonging to the curriculum of Metallurgical Civil Engineering and Materials at the Technical University Federico Santa María, Valparaiso, Chile. The objectives of the research were to determine the most important aspects of the course that required methodological intervention. This resulted in a curricular remodeling that was consistent with the educational model declared by the university. This was then evaluated through the students' perceptions, once the innovations were implemented. The UTFSM educational model seeks to allow students to learn from their experience, permitting them to reflect and develop a critical attitude towards their actions and decisions. In concordance, the innovations implemented corresponded to active methodologies, based on the constructivist model. Students through group activities, individual reflection, plenary discussion and use of thermodynamic software developed an understanding about the effects of operation variables on metal production processes. For the development and implementation of the methodologies, - during the years 2015 and 2016 of master classes - teacher evaluation surveys were regularly conducted to students of pyrometallurgy courses with self-applied guidelines, where they were asked to express their opinions on their learning experience. They could refer to criticisms, improvements and suggestions around the methodology, course content and teaching work.

The students' opinions were analyzed through an evaluation of the content of discourse, within a descriptive exploratory qualitative research design and through quantitative questions. These surveys made it possible to identify learning needs, brought the course's design process and assessment methodology closer to students. The active methodologies, defined from the analysis of the responses given by the students, were implemented during the first half of 2019. This implementation was monitored with surveys similar to those used in 2015 and 2016, where content analysis was also utilized. The results of the present investigation showed that the innovative approach of the course allowed for students to become active participants in the structuring it's design and methodology. This was consistent with the educational model of the university and the 


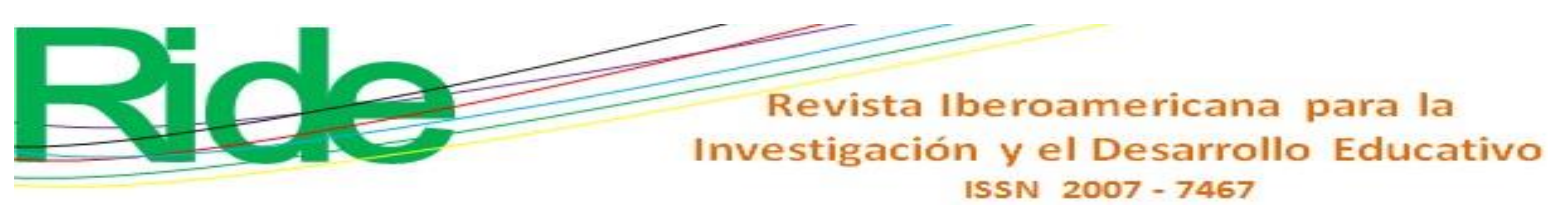

rationalization of the course content. Student's perceptions regarding content communication, teaching environment and learning level improved significantly regarding the courses given with the master classes. The results indicated that the active methodologies contributed effectively to the participation of students in class, promoted dialogue in group activities and created better learning conditions.

Keywords: methodological innovation, active learning methodology, constructivist model, pyrometallurgy, course redesign.

\section{Resumo}

Este trabalho descreve uma inovação na metodologia de ensino da disciplina de Pyrometallurgy, pertencente ao currículo da carreira de Engenharia Civil Metalúrgica e de Materiais da Universidade Técnica Federico Santa María (UTFSM), Valparaíso, Chile. Os objetivos da pesquisa foram determinar os aspectos mais importantes do curso que exigiam uma intervenção metodológica. Isso resultou em um redesenho curricular consistente com o modelo educacional declarado pela universidade. Isso foi avaliado através da percepção dos alunos, uma vez que as inovações foram implementadas. O modelo educacional da UTFSM busca permitir que os alunos aprendam com sua experiência, permitindo que eles reflitam e desenvolvam uma atitude crítica em relação a suas ações e decisões. De acordo, as inovações implementadas corresponderam a metodologias ativas, baseadas no modelo construtivista. Os alunos - por meio de atividades em grupo, reflexão individual, discussão em plenária e uso de software termodinâmico desenvolveram aprendizado sobre os efeitos das variáveis operacionais nos processos de produção de metal. Para o desenvolvimento e implementação das estratégias, durante os anos de 2015 e 2016 (cursos correspondentes à modalidade de aulas de mestrado), foram realizadas regularmente pesquisas de avaliação de professores em alunos com diretrizes autoaplicáveis, nas quais foi solicitado que expressassem suas opiniões sobre sua experiência de aprendizagem. Eles poderiam encaminhar críticas, bem como melhorias em torno da metodologia, do conteúdo do curso e do trabalho de ensino. As opiniões registradas foram analisadas por meio da análise do conteúdo do discurso, no delineamento de pesquisa qualitativa exploratória descritiva e por questões de natureza quantitativa. Essas pesquisas possibilitaram identificar necessidades de aprendizagem, aproximaram o desenho do curso e a metodologia de avaliação dos alunos. As metodologias ativas definidas a partir da análise das respostas dadas pelos alunos; Eles foram implementados durante o primeiro semestre de 2019. Essa implementação foi monitorada com pesquisas semelhantes às 


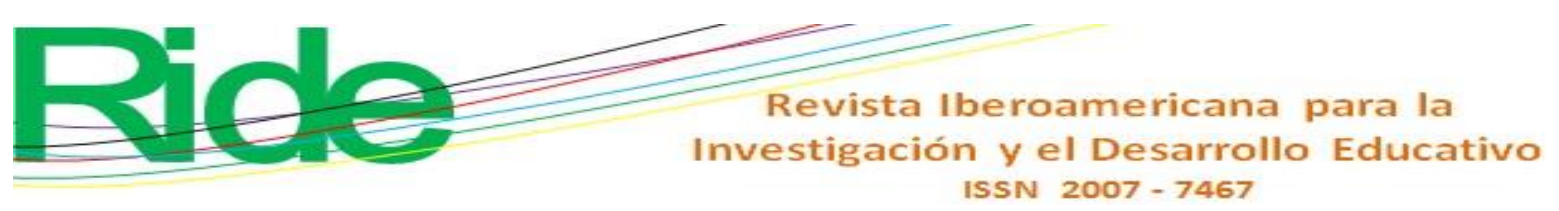

utilizadas em 2015 e 2016, onde também foi usada a análise de conteúdo. Os resultados da presente investigação mostraram que a abordagem inovadora do curso; permitiu que os alunos se tornassem participantes ativos na estruturação de seu design e metodologia. Isso foi consistente com o modelo educacional da universidade e a racionalização do conteúdo do curso. As percepções dos alunos sobre a comunicação dos conteúdos, o ambiente de ensino e o nível de aprendizagem melhoraram significativamente em comparação aos cursos ministrados nas aulas de mestrado. Os resultados indicaram que metodologias ativas contribuíram efetivamente para a participação dos alunos nas aulas, promoveram o diálogo nas atividades em grupo e criaram melhores condições de aprendizado.

Palavras-chave: inovação metodológica, metodologia de aprendizado ativo, modelo construtivista, pirometalurgia, redesenho de curso.

Fecha Recepción: Septiembre 2019

Fecha Aceptación: Abril 2020

\section{Introducción}

En el marco contextual del modelo educativo de la Universidad Técnica Federico Santa María (UTFSM) (2015) se declara lo siguiente:

Los constantes cambios y transformaciones de la sociedad actual desafían a las instituciones de Educación Superior a formar líderes integrales, profesionales y graduados competentes, a través de una propuesta educativa de excelencia que prepare a sus egresados para el ámbito humano, científico técnico y profesional. Lo que se logra desarrollando aprendizajes desde la experiencia de los estudiantes, que le permitan reflexionar y tener conciencia crítica frente a sus acciones y decisiones (p. 12).

Esta consigna se encuentra en sintonía con los principales fundamentales del modelo constructivista explicado por Ausubel, Novak y Hanesian (1983) e Irzik (2000), según el cual el proceso de aprendizaje se apoya en los conocimientos previos que se tengan sobre determinado tema, así como en la actividad externa o interna que el estudiante realice al respecto. Para facilitar esa tarea, por supuesto, resulta indispensable el apoyo que pueda brindar un agente externo, el cual es desempeñado por el docente en el sistema educativo formal. Esa participación es descrita en el referido modelo educativo de la UTFSM del siguiente modo: 


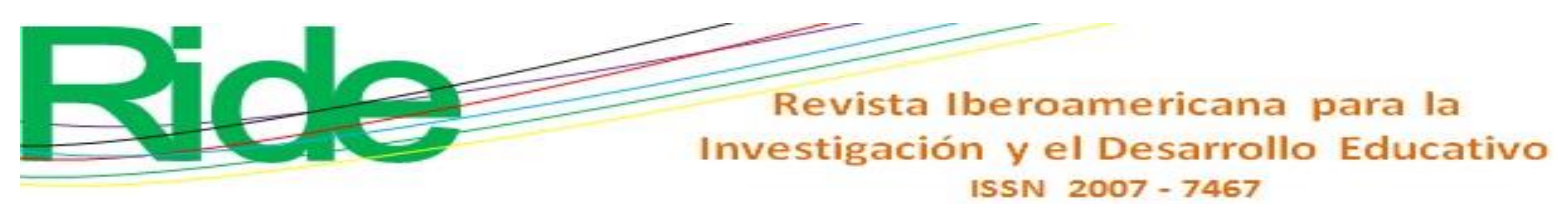

La actividad docente es un factor esencial en este proceso, guiando y apoyando la gestión del conocimiento donde el rol del estudiante es central. Se entienden los roles del profesor, del instructor y del ayudante de docencia como moderadores de los procesos de enseñanza-aprendizaje; actores esenciales en permanente perfeccionamiento, tanto en los aspectos metodológicos para la formación docente como en su disciplina, a fin de generar las mejores condiciones para el desarrollo integral de los estudiantes (p. 14).

Ahora bien, intentado establecer una coherencia entre la acción pedagógica de aula y el citado modelo educativo de la UTFSM, en el presente trabajo se intenta describir un proceso de innovación en lo referido a la enseñanza de la cátedra Pirometalurgia con metodologías activas de aprendizaje. El propósito es procurar que los estudiantes de Ingeniería Civil Metalúrgica que cursan dicha asignatura se apropien de los conceptos termodinámicos necesarios para entender, aplicar y proyectar cualquier cambio en los procesos productivos.

Un antecedente enfocado en la enseñanza de la termodinámica en la carrera de ingeniería empleando metodologías activas es el trabajo de Espinoza, Silva y López (2017), quienes se apoyaron en los postulados del aprendizaje significativo de Ausubel et al. (1983) para influir positivamente en el rendimiento y en el desarrollo de competencias de los estudiantes. Según estos autores, esa iniciativa surgió porque detectaron que a estudiantes de un curso de termodinámica de la carrera Ingeniería Civil Industrial se les dificultaba aplicar los conocimientos adquiridos a problemas reales, es decir, "no lograban "usar un análisis correcto y minucioso de las situaciones dadas en cada experimento o situación planteada" (Espinoza et al., 2017, p. 3).

Las preguntas planteadas para el desarrollo del presente trabajo fueron las siguientes: ¿es posible mejorar la percepción de los estudiantes acerca del curso de Pirometalurgia con el uso de metodologías activas? ¿Se pueden adaptar algunas metodologías activas de enseñanza-aprendizaje de ciencias básicas (por ejemplo, física y termodinámica) en un área de ingeniería aplicada como la pirometalurgia?

La hipótesis propuesta fue que las metodologías activas de aprendizaje no solo se pueden empelar en el área de la pirometalurgia, sino que también pueden impactar positivamente en la percepción de los estudiantes en cuanto los procesos de comunicación de contenidos, ambiente de enseñanza y nivel de aprendizaje fomentados en el curso. 


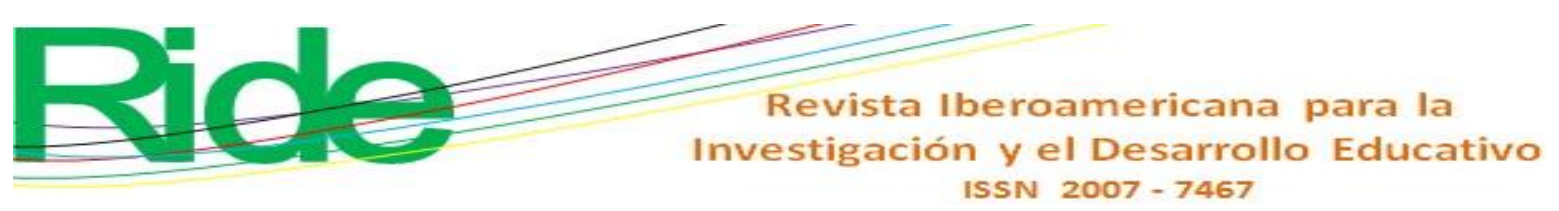

Los objetivos planteados fueron los siguientes:

a. Determinar los aspectos más destacables del curso aplicado, para lo cual se emplearon encuestas de evaluación docente con pautas autoaplicadas. Algunas preguntas formuladas tuvieron una naturaleza cuantitativa, aunque también se recabaron opiniones de los estudiantes que fueron analizadas a través de un análisis del discurso.

b. Efectuar un rediseño curricular coherente con el modelo educativo constructivista declarado por la UTFSM, para lo cual se emplearon metodologías activas de enseñanza/aprendizaje.

c. Validar o refutar la hipótesis planteada anteriormente mediante la evaluación de la percepción de los estudiantes.

\section{Metodología}

\section{Descripción del curso de Pirometalurgia}

En el perfil de egreso del ingeniero civil metalúrgico de la UTFSM se declara lo siguiente: El ingeniero civil metalúrgico UTFSM es un profesional con base científica, especializado en las tecnologías de extracción, procesamiento, aplicación y reciclado de metales, para satisfacer las necesidades industriales y de la sociedad en general. Su formación en ciencias básicas y ciencias de ingeniería le permite resolver problemas equivalentes en otras de materiales. El campo de ejercicio de la profesión comprende: la operación de instalaciones y procesos industriales; el diseño y proyecto de plantas de proceso; el soporte técnico en la comercialización de tecnologías, productos y materiales; los servicios especializados de ingeniería; y la investigación y desarrollo de nuevos productos, procesos y aplicaciones. En cada uno de estos ámbitos aplica un conjunto distintivo de conocimiento matemático, científico e ingenieril a los problemas metalúrgicos, en una perspectiva de desarrollo sustentable. Para plantear y resolver problemas combina el uso de modelos, propio de la ingeniería de procesos, con el diagnóstico sintomático, propio de la ingeniería de materiales (UTFSM, 2013, parr 1-3).

El ingeniero civil metalúrgico como especialista en la transformación de minerales en materiales para la vida cotidiana tiene grandes desafíos, entre los que se destacan los siguientes: enfrentar con innovación la creciente disminución de minerales, energía y agua y desarrollar materiales reciclables a bajos precios que cumplan los estándares de calidad requeridos sin dañar el medio ambiente. 


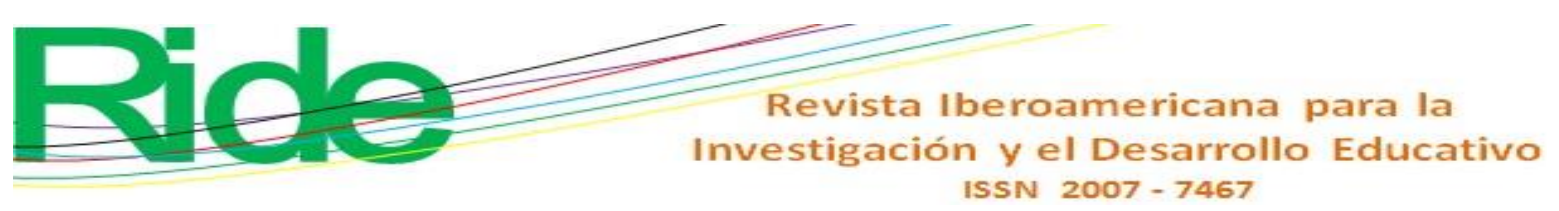

En este contexto, en el presente trabajo se describe un proceso de enseñanza basado en metodologías activas para la asignatura Pirometalurgia., la cual se imparte en el noveno semestre del plan de estudios de la carrera Ingeniería Civil Metalurgia y Materiales (UTFSM, 2017, p. 4). La cantidad de estudiantes que cursan esta asignatura suele ser entre 20 y 30 personas, las cuales debieron haber aprobado materias como Mecánica de Fluidos, Termodinámica Metalúrgica I y II, Transferencia de Calor, y Cinética y Diseño de Reactores Metalúrgicos.

Los contenidos temáticos de Pirometalurgia se pueden representar según el esquema indicado en la figura 1. Esta cátedra se halla relacionada con la producción y el reciclaje de metales, entre los que se pueden mencionar el cobre, el níquel, el plomo y el zinc. Los procesos pirometalúrgicos están fundamentados en ciencias básicas de la ingeniería como termodinámica, cinética y fluodinámica. Durante las clases se intenta promover una interacción de aprendizaje entre los procesos pirometalúrgicos y los conceptos termodinámicos, donde los procesos metalúrgicos son asimilados por medio de estos conceptos. El porqué de los procesos se comprende a través del uso de diagramas de fases relacionados con las presiones parciales de los gases (oxígeno, azufre, arsénico) presentes en las reacciones químicas de los casos analizados. Asimismo, se incluyen elementos específicos de aplicación - como la teoría de soluciones y otros más indicados en la figura 1 - que permiten efectuar evaluaciones energéticas, ambientales y otras. El curso está diseñado para total de 166 horas por semestre, de las cuales 66 se consideran como trabajo no presencial que debe cumplir el estudiante. 


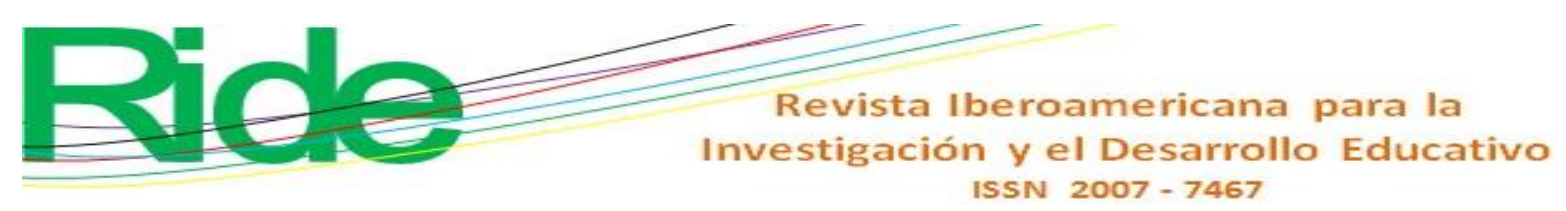

\section{Mejoras en torno al material de apoyo: 13 frases}

Respecto al material de apoyo empleado en las charlas magistrales utilizando power point, los participantes indicaron que se debían efectuar mejoras en torno al diseño y su uso. En relación con el diseño, apuntaron que al material de apoyo le faltaba orden, de ahí que recomendaran que fuera reelaborado para ser usado como material de estudio posterior.

\section{Mejoras relativas a las instrucciones y las actividades: $\mathbf{2 6}$ frases}

Algunas menciones se vincularon con la calidad de las actividades. Destacaron el valor que se otorgó a los procesos traducidos en diagramas y ejercicios, además del detalle con que estaban tratados los parámetros. Fue ponderada positivamente la forma de evaluar y los trabajos realizados. En esta categoría, el discurso se refirió a un bajo nivel de instrucción, y no a su ausencia. Igualmente se refirió el favorecer el orden en las explicaciones dadas.

En cuanto a las actividades propuestas, sugirieron una mejor coordinación de las ayudantías, laboratorios y clases a través de un cronograma propuesto desde el inicio del semestre. También recomendaron una mayor orientación del profesor a quienes ejecutaban las ayudantías para contribuir más eficazmente a los objetivos del curso. Además, enfocar las ayudantías en optimizar la preparación previa de los estudiantes a los certámenes y fortalecer los ejercicios con calculación numérica.

\section{Mejoras sobre la explicación y cualidades pedagógicas del profesor: 26 frases}

Los estudiantes señalaron que el profesor realizó explicaciones adecuadas y pertinentes con el quehacer. Destacaron el manejo de la materia y el dominio de contenidos junto con la capacidad de transmitirlos. Al demostrar dominio específico de conceptos complejos, se incentivó la motivación por el curso.

Igualmente, expresaron que hubo una actitud receptiva a las dudas de los estudiantes tanto en el aula como fuera de esta. También señalaron que el profesor estaba dispuesto a responder preguntas en todo momento. Destacaron la disposición del profesor y su interés para que el estudiante aprendiera. Finalmente, los participantes consideraron que fue potenciado el trabajo en equipo e investigativo.

Los estudiantes, sin embargo, opinaron que el docente podría mejorar el cierre de las clases, con conclusiones más explícitas, escritas en la pizarra. Paralelo al análisis de variables 


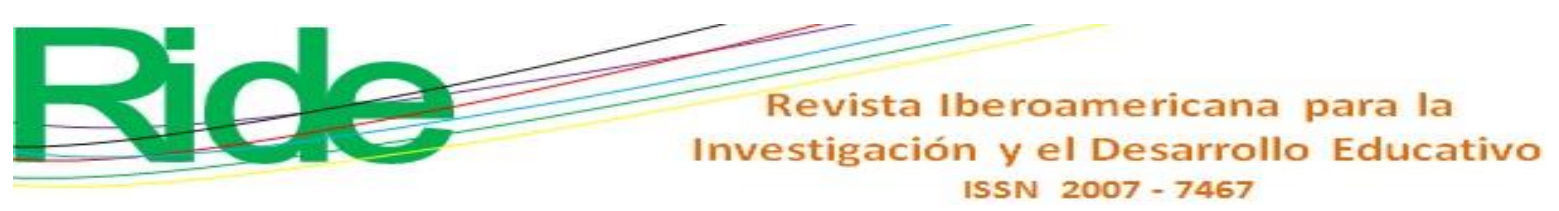

termodinámicas de los casos estudiados en clase, el profesor podría desarrollar calculaciones numéricas que representen estos análisis.

\section{Sugerencias metodológicas: $\mathbf{4 0}$ frases}

La mayor frecuencia de frases se refirió a las mejoras en torno a la propuesta metodológica. $\mathrm{Al}$ respecto, apuntaron que se podría modificar la dinámica de la clase, aumentarla y cambiar de enfoque. También recomendaron hacer más énfasis en los análisis matemáticos, así como una mayor ejemplificación de los casos. Igualmente, se pidió clarificar el sistema de evaluación.

En cuanto a la metodología, señalaron mayor discusión con participación de todo el curso, y no por separado. Además, indicaron la necesidad de revisar el excesivo tiempo empleado en la revisión de tutoriales y la necesidad de aproximarse a situaciones reales a través de ejercicios y ejemplos industriales y programar visitas a una fundición. También mencionaron que se debía efectuar una retroalimentación sobre los certámenes y los errores en la ejecución de tutoriales, así como mejorar el orden en la exposición de la información. Además, sugirieron más planificación del curso y nivelar los conocimientos de termodinámica al comienzo del curso.

Por otra parte, hubo aspectos de la metodología que fueron ponderados positivamente, tal fue el caso de los tutoriales y las charlas. Explicitaron que la información fue entregada en forma secuencial y lógica, e indicaron que durante el curso se complementaron los contenidos y a través de los tutoriales se explicitó el aprendizaje.

\section{Discusión acerca de las encuestas de evaluaciones docentes 2016}

La innovación metodológica descrita en este trabajo procuró exponer las debilidades, gestionar las fortalezas y las propuestas indicadas por los estudiantes en la evaluación docente e intervenir el curso con elementos que permitieran un proceso constructivista de enseñanza y aprendizaje.

Los resultados de las encuestas cuantitativas del curso 2016 (vale aclarar que en el año 2015 no se efectuaron preguntas de esta índole) permitieron detectar algunas deficiencias en cuanto al curso. Para la evaluación docente, se empleó una escala de 0 a 4 (en este rango se consideró que 3 sería una calificación deficiente), está se muestra en la figura 2. El nivel de aprendizaje alcanzado por los estudiantes fue clasificado de 1 a 7 (una calificación de 4.5 fue estimada como deficiente), es mostrado en la figura 3. En síntesis, se puede decir que el curso de Pirometalurgia tuvo un resultado substancialmente por debajo del promedio del total de la UTFSM. 


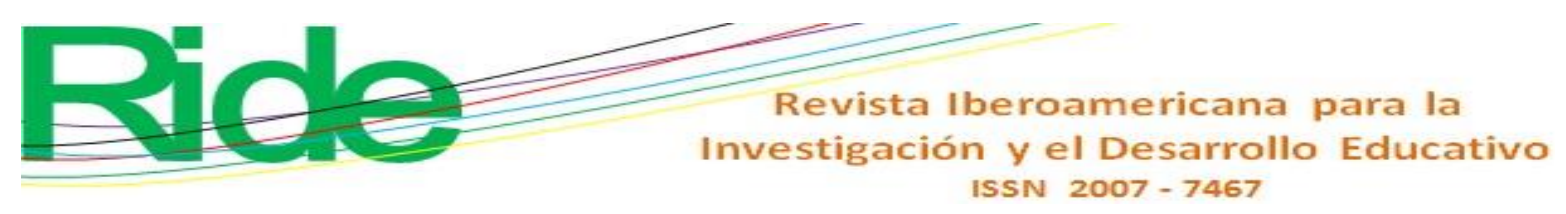

\section{Rediseño del curso en metodología y evaluación con un enfoque constructivista}

\section{Replanteamiento de las charlas magistrales}

El propósito de esta actividad de intervención fue pasar de un esquema de transmisión de información a uno con métodos de enseñanza activos (Mills y Treagust, 2003), como se indica en la tabla 1. En énfasis se hizo en el diseño del curso para ayudar al estudiante a aprender de forma distinta a como lo hace con el método tradicional, el cual se centra en la trasmisión de conocimientos (Mazur, 2009). El objetivo de las charlas fue promover conflictos conceptuales que le permitieran al estudiante conceptualizar los procesos desde nuevas perspectivas (Hewson y Beckett, 1984).

Tabla 1. Comparación de los dos métodos de enseñanza

\begin{tabular}{|l|l|}
\hline Método anterior & Método actual \\
\hline $\begin{array}{l}\text { Énfasis: qué enseñar y qué } \\
\text { libro usar. }\end{array}$ & $\begin{array}{l}\text { Énfasis: Cómo enseñar, en especial cómo ayudar a los } \\
\text { estudiantes a aprender. }\end{array}$ \\
\hline $\begin{array}{l}\text { Presentaciones para de } \\
\text { entregar información } \\
\text { (transmisión } \\
\text { información). }\end{array}$ & $\begin{array}{l}\text { Curso basado en técnicas de aprendizaje y enseñanza activos. } \\
\text { Promover el pensamiento en términos de conceptos básicos } \\
\text { (termodinámica, cinética y fluohidrodinámica). } \\
\text { Énfasis en el uso de herramientas de simulación y casos de } \\
\text { estudio. }\end{array}$ \\
\hline $\begin{array}{l}\text { Enseñanza basada en } \\
\text { presentaciones. }\end{array}$ & Enseñanza basada en debate y trabajos grupales. \\
\hline $\begin{array}{l}\text { Amplia cobertura de } \\
\text { temas-baja comprensión. }\end{array}$ & $\begin{array}{l}\text { Menos cubrimiento de temas-alta comprensión. } \\
\text { Presentación breve de los tópicos. }\end{array}$ \\
\hline
\end{tabular}

Fuente: Elaboración propia

\section{Rediseño de la didáctica de cada tópico específico}

En la figura 4, se esquematiza la didáctica diseñada empleando tres círculos concéntricos que indican la secuencia a enseñar de un tópico específico (el de secado). El primer círculo indica las evidencias de desempeño que se pueden lograr en resultados de aprendizaje (RdA); el segundo se refiere a las herramientas que se deben utilizar para lograr los resultados de aprendizaje, y el tercero el método de evaluación. Las herramientas de simulación fueron el soporte para el cómo (Lewis y Linn, 2003), para lo que se emplearon programas de simulación comerciales. La simulación fue asociada a comparar los procesos con diferentes parámetros de entrada como casos de estudio. Los 


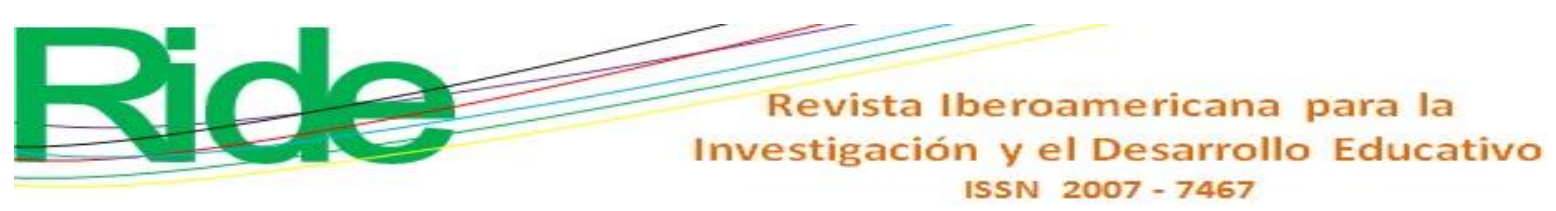

resultados de estas simulaciones permitirán en parte la discusión argumentada en resultados (Martini y Hartzell, 2015). Externamente, se indican los factores adicionales para evaluar el éxito o las deficiencias del proceso de enseñanza y aprendizaje.

Figura 4. Diseño didáctico de un tópico específico

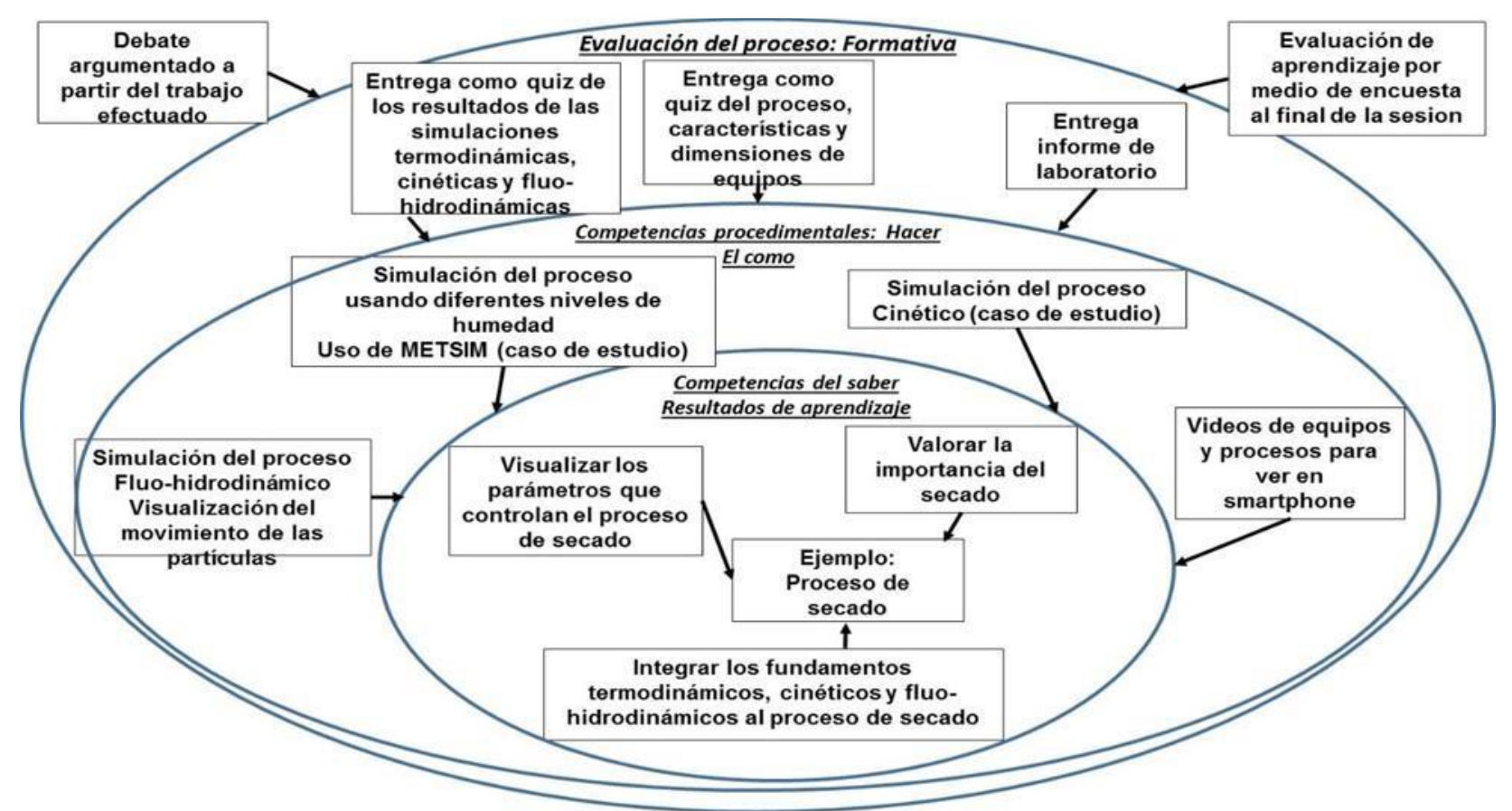

Fuente: Elaboración propia

\section{Innovación en el diseño de la secuencia didáctica de cada clase}

Consonante con lo anterior, fueron realizadas las innovaciones metodológicas a través de la planificación didáctica. Esta consistió en proponer actividades de aprendizaje activo, como el debate y la integración de las tecnologías como herramientas de apoyo para el aprendizaje. En el aula, la sesión fue de una hora y media (dos sesiones por semana). La secuencia didáctica de una sesión se indica en la figura 5, donde se observa cómo las intervenciones de presentaciones magistrales no tienen una duración mayor a 10 minutos y las actividades las efectúan en su mayor parte los estudiantes a través de trabajos de simulación: uso de información termodinámica para descubrir el porqué de los procesos (Hewson y Beckett, 1984), discusión grupal argumentada basada en los resultados de simulación y utilización de elementos tecnológicos como parte integral para describir los procesos. 


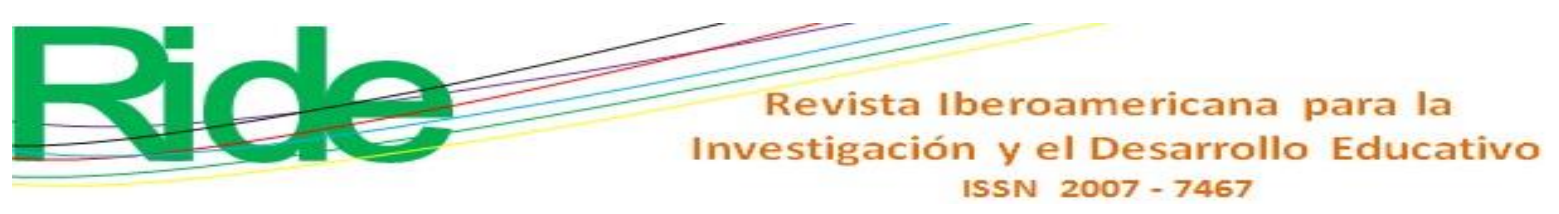

Figura 5. Secuencia didáctica de una sesión

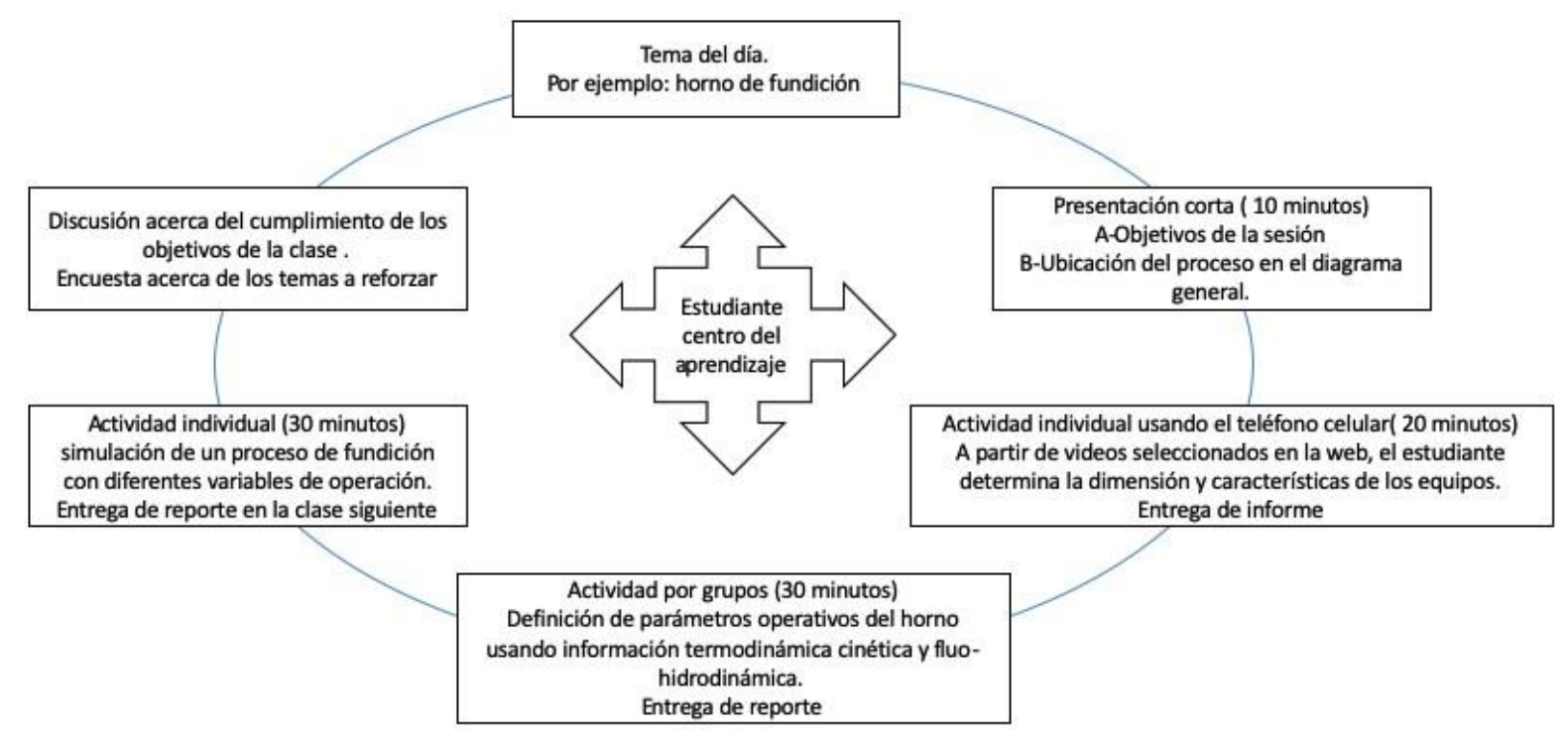

Fuente: Elaboración propia

\section{Replanteamiento del sistema de evaluación}

Para el diseño de esta innovación se buscó consistencia entre los tres componentes del diseño curricular (Cowan y Cherry, 2012), como se indica en la figura 6.

Figura 6. Interacción entre los tres componentes principales del diseño de un curso

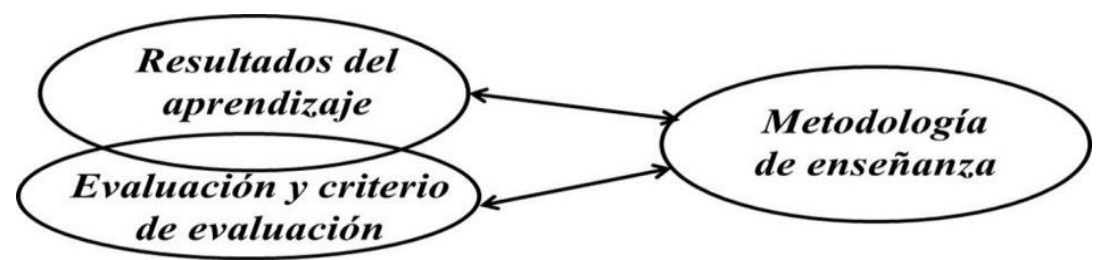

Fuente: Cowan y Cherry (2012)

El principio fundamental fue lograr que los tres componentes estuvieran diseñados para responder a las siguientes tres preguntas:

1. ¿Qué evaluaciones y qué criterios de evaluación utilizará para mostrar que los estudiantes han logrado los resultados de aprendizaje? Esto fue abordado por una evaluación participativa de los estudiantes de todas las clases junto a una retroalimentación de resultados permanente. Asimismo, esto incluyó la socialización de planes para implementar mejoras. 


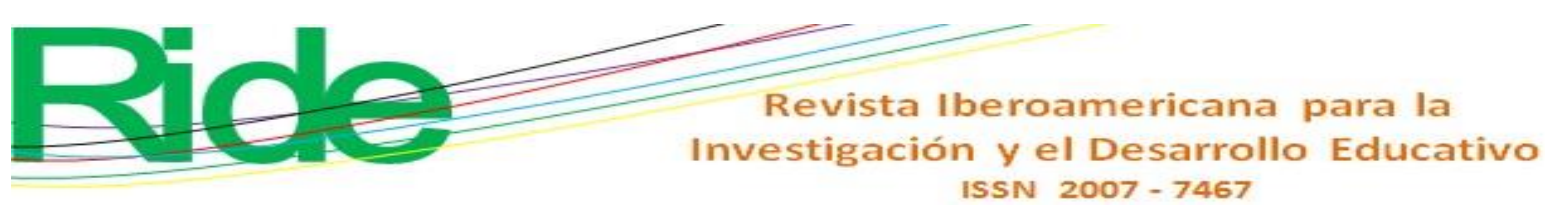

2. ¿Qué métodos de enseñanza utilizará para lograr dichos resultados de aprendizaje?

3. ¿Qué desea que su estudiante aprenda? Para esto, se tuvo en cuenta el perfil de egreso declarado por el departamento, el cual indica, entre otros aspectos, los siguientes:

- Aplicar las ciencias básicas y las ciencias de la ingeniería que sustentan la ingeniería metalúrgica.

- Comunicarse con otras personas en forma oral y escrita.

- Participar como integrante y posteriormente como conductor en equipos de trabajo.

Asimismo, como resultados de aprendizaje se espera que el estudiante logre lo siguiente:

- Aplicar los fundamentos teóricos utilizados en los procesos pirometalúrgicos.

- Adquirir conocimientos básicos de los procesos unitarios empleados en la pirometalurgia.

- Correlacionar cada proceso unitario describiendo el proceso general.

- Transferir un proceso específico a otro, aplicando procedimientos de la industria.

- Analizar los consumos energéticos e impactos ambientales, considerándolos en la cadena de producción y reciclaje.

Las evaluaciones sumativas y formativas se muestran en la tabla 2, la cual incluye la contribución al perfil de egreso del Departamento de Ingeniería Metalúrgica y de Materiales (UTFSM, 2013). Se espera con dichas evaluaciones abordar la interacción indicada en la figura 4 y los resultados de aprendizaje trazados. 


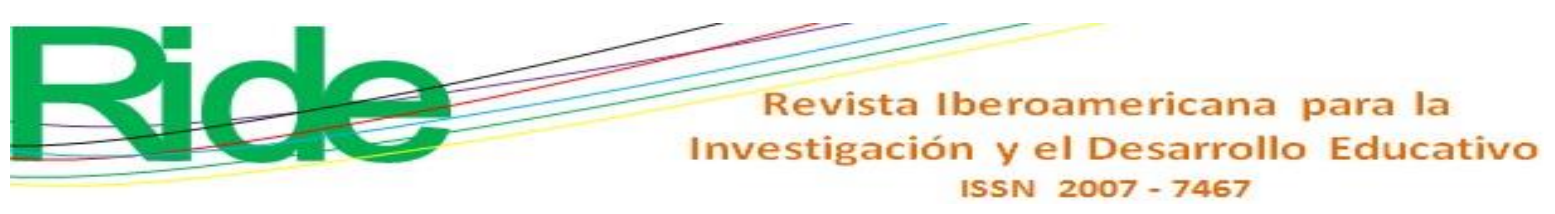

Tabla 2. Plan de evaluación formativa y sumativa de la asignatura

\begin{tabular}{|l|l|l|}
\hline Evaluación del curso & Peso & $\begin{array}{l}\text { Contribución } \\
\text { al perfil de } \\
\text { egreso }\end{array}$ \\
\hline Académica & $\mathbf{1 5}$ & $\mathbf{1 , 2}$ \\
\hline $\begin{array}{l}\text { 1-Tutoriales total = 7; valor de cada tutorial 2 } \\
\text { puntos }\end{array}$ & $\mathbf{1 5}$ & $\mathbf{1 , 2 , 3}$ \\
\hline $\begin{array}{l}\text { 2-Balance de masa y energía (reporte del HSC) } \\
\text { trabajo acumulado durante el semestre }\end{array}$ & $\mathbf{1 0}$ & $\mathbf{1 , 2}$ \\
\hline $\begin{array}{l}\text { 3-Lectura, reporte y presentación de un } \\
\text { documento (cinco minutos de presentación) }\end{array}$ & $\mathbf{1 0}$ & $\mathbf{1 , 2 , 3}$ \\
\hline $\begin{array}{l}\text { 4- Construcción e interpretación de diagramas de } \\
\text { fase ternario de uso en la pirometalurgia (trabajo } \\
\text { en grupo) }\end{array}$ & $\mathbf{1 5}$ & $\mathbf{1}$ \\
\hline $\begin{array}{l}\text { 5- Certamen intermedio (nota mínima 40 en una } \\
\text { escala de 100) }\end{array}$ & $\mathbf{1 5}$ & $\mathbf{1}$ \\
\hline $\begin{array}{l}\text { 6-Certamen final (nota mínima 50 en una escala } \\
\text { de 100) }\end{array}$ & $\mathbf{8 0}$ & $\mathbf{2 0}$ \\
\hline Total & $\mathbf{1 , 2 , 3}$ \\
\hline Laboratorios & & \\
\hline Reportes de laboratorio & & \\
\hline
\end{tabular}

Fuente: Elaboración propia

\section{Resultados}

\section{Implementación de las innovaciones educativas en el primer semestre 2019}

Las innovaciones implementadas fueron evaluadas al final de cada clase, incluyendo tres preguntas en las cuales se les solicitó a los estudiantes que escribieran brevemente sus opiniones sobre de lo siguiente:

1. Lo que el instructor realizó especialmente bien durante la clase.

2. Lo que el estudiante creía que se debería mejorar.

3. Lo qué aprendió.

En total, participaron 23 estudiantes, respectivamente, los cuales redactaron un total de 129 frases. Las siguientes fueron las categorías definidas, aunque vale destacar que algunas respuestas similares pueden aparecer en categorías diferentes. 


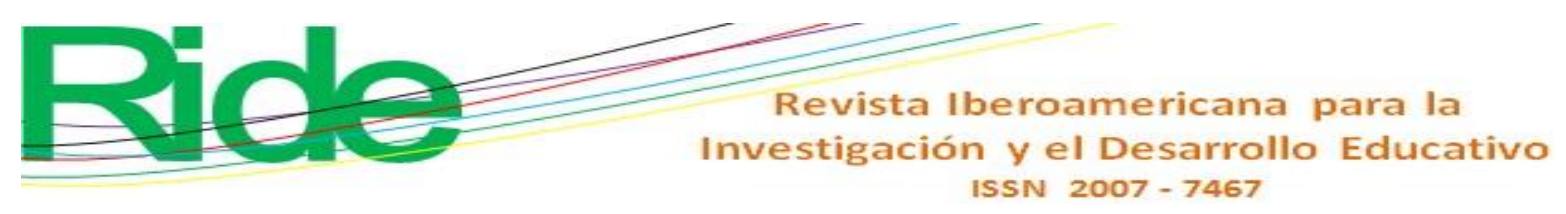

Mejoras sugeridas a la metodología: 31 frases

En cuanto al material de estudio, propusieron aumentar el estudio de situaciones industriales reales para facilitar el aprendizaje, así como aumentar la dinámica de la clase a través de la discusión de los tópicos con preguntas más reflexivas y mayor participación individual y colectiva. Asimismo, recomendaron aumentar el material de apoyo educativo (en esta categoría se mencionaron videos, diapositivas, diagramas de fases y tutoriales). Vale comentar que se presentaron dificultades con los enlaces ofrecidos para acceder a los videos, por lo que sugirieron que estos se debían entregar antes de la clase o facilitar el nombre para buscarlos en la Web. Además, mencionaron que se debía mantener el esquema de diapositivas y el estudio de diagramas de fases. Respecto a los tutoriales, propusieron que se debía ofrecer una explicación con más detalle sobre cómo hacerlos y establecer un calendario de entregas.

Respecto a la metodología utilizada, consideraron que no se debería avanzar tan lento, por lo que se debía aumentar el tiempo para la explicación conceptual. Asimismo, al final de la discusión grupal, el profesor debería terminar con una conclusión y profundizar más en lo aprendido a través de ejercicios con calculaciones numéricas, y no solo con el análisis de las variables termodinámicas de los procesos pirometalúrgicos.

\section{Mejoras sugeridas a la explicación y cualidades pedagógicas del profesor: 51 frases}

La presencia de frases en torno a explicaciones claras y concisas del profesor sobre conceptos fue significativa (uso adecuado del tiempo, contenidos explicados en forma llamativa, clases desarrolladas en forma dinámica que crearon un ambiente de aprendizaje ameno). En otras palabras, se apreció orden y buen contenido en las presentaciones con explicaciones didácticas, de ahí que se infiera que la transmisión de la información fue eficaz y que la interacción fue promovida de forma adecuada. Aunado a esto, el uso de los videos constituyó un recurso muy ilustrativo.

El profesor generó espacios de discusión a partir de problemas reales en los procesos industriales y promovió en los estudiantes el pensamiento crítico para buscar explicaciones. Asimismo, los motivó para que ellos emitieran sus opiniones e interactuaran con los compañeros. Guio a los grupos para que obtuvieran sus propias respuestas y aumentó la dinámica de las discusiones formulando más preguntas para profundizar el nivel de conocimiento de los estudiantes. En síntesis, el profesor fungió como un moderador (no como alguien que solo dicta materia). 


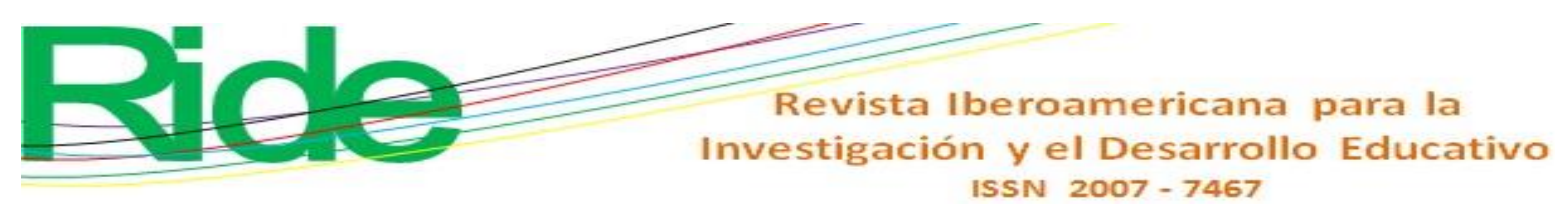

Las sugerencias de mejoras, sin embargo, se enfocaron en que el profesor podría ofrecer un resumen en la pizarra de los conceptos y de consideraciones que los estudiantes debían entender y hacer de las variables termodinámicas (en los casos de estudio de procesos pirometalúrgicos tratados en clase), así como de sus calculaciones numéricas.

\section{Nivel de aprendizaje referido: 47 frases}

La respuesta de los estudiantes respecto a los aprendizajes referidos se enfocó en la importancia del análisis de gráficos para la construcción de un conocimiento acerca del mercado mundial del cobre. Otro aspecto puntual fue la generación de conciencia sobre el daño ambiental causado por elementos que hacen parte de los procesos pirometalúrgicos tales como el arsénico.

Por otra parte, aunque de forma más general, se valoró que durante las clases se hubiera logrado una interacción de aprendizaje entre los procesos pirometalúrgicos y los conceptos termodinámicos, aspecto esencial para asimilar los procesos metalúrgicos. De hecho, los procesos pirometalúrgicos fueron comprendidos a través de gráficos (diagramas de fases) relacionados con la termodinámica. Además, indicaron que se comprendió el porqué de los procesos a través del uso de diagramas de fases relacionados a las presiones parciales de los gases (oxígeno, azufre, arsénico) presentes en las reacciones químicas planteadas.

\section{Encuesta final del curso en el primer semestre 2019}

Al finalizar el semestre, se aplicó una encuesta final en la cual participaron 15 estudiantes; en esta se incluyeron las mismas tres preguntas de la encuesta descrita anteriormente y fueron analizadas del mismo modo.

\section{Aspectos positivos de la experiencia educativa: 48 frases}

Las respuestas de los estudiantes se clasificaron de acuerdo con los cambios metodológicos indicados en la tabla 1. En cuanto al criterio cómo enseñar, los participantes consideraron que el curso fue bien diseñado y organizado, según el orden en que los procesos son estudiados (se inició secuencialmente de lo más fácil a lo más complejo), y opinaron que las clases fueron prácticas y didácticas.

En cuanto al curso basado en técnicas de aprendizaje y enseñanza activas y promover el pensamiento en términos de conceptos básicos, los estudiantes opinaron que los contenidos fueron explicados con muchos ejemplos, demostraciones prácticas, experimentales y simulación, lo que 


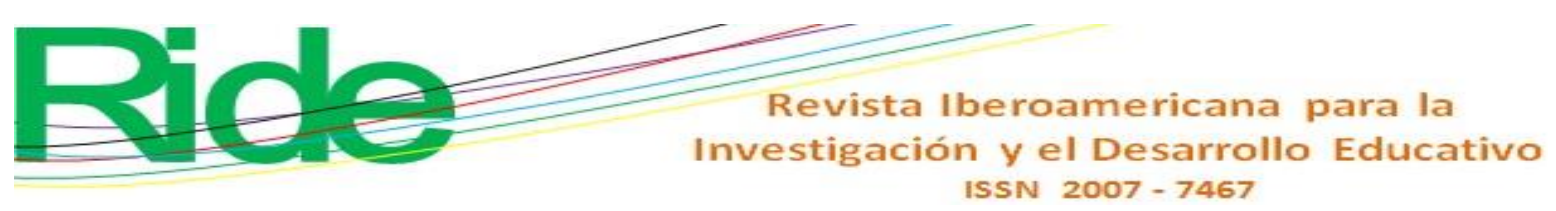

sirvió para analizar y profundizar en los aspectos termodinámicos involucrados en los procesos pirometalúrgicos estudiados. Los laboratorios fueron una buena complementación al curso. Consideraron que se estableció una relación clara entre la termodinámica y los procesos de pirometalurgia. También manifestaron que aprendieron a relacionar los procesos de extracción de cobre con los de otros minerales, así como los diagramas de fases ternarias y binarias teóricas y/o experimentales con la práctica. Además, comprendieron la importancia del control de parámetros en cada proceso dependiendo de lo que se buscaba obtener.

Sobre la enseñanza basada en debate y trabajos grupales, las opiniones de los estudiantes incluyeron que el curso fomentó el trabajo colaborativo a través de análisis del material de estudio en grupos. El método empelado, además, les permitió plantear cuestionamientos interesantes para profundizar en los contenidos.

\section{Mejoras referidas a la experiencia educativa: 27 frases}

Las respuestas de los estudiantes a las mejoras educativas han sido clasificadas en esta sección tomando en cuenta el diseño didáctico sobre las competencias de saber y procedimentales, así como la evaluación formativa indicada en la figura 4. Respecto a las competencias del saber, los estudiantes sugirieron que el curso debía incluir una revisión de los conceptos termodinámicos durante las dos primeras sesiones de clase. Según sus percepciones, el curso tendría que complementarse con una sesión de clase de fundamentos teóricos y dedicar la siguiente sesión al estudio de procesos y a consultas. Aunado a esto, consideraron que en cada clase se podría establecer lo que se quería analizar en los procesos estudiados. Igualmente, propusieron extender los temas de estudio para los procesos pirometalúrgicos que no se vinculen con el cobre y complementar el curso con salidas a terreno.

En lo concerniente a las competencias procedimentales, indicaron que las guías utilizadas como material de trabajo en clase se debían entregar de forma individual, y no grupal. También consideraron que se perdía tiempo en la búsqueda de los videos y que se presentaba un desfase entre las distintas reproducciones. Por tanto, coincidieron en que los videos deberían ser presentados para todo el grupo, y no revisados individualmente en el teléfono celular.

Sobre la evaluación del proceso, señalaron que cuando concluía el análisis de un proceso, el profesor podría explicitar en la pizarra las conclusiones obtenidas e incrementar las calculaciones en los procesos analizados. Además, indicaron que el curso demandaba mucho tiempo de estudio extra clase, por lo que recomendaron aumentar los créditos asignados. 


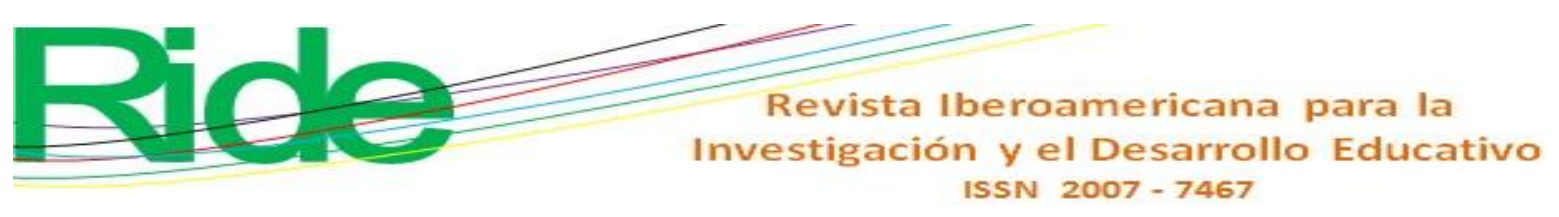

\section{Comparación de la evaluación docente del curso de Pirometalurgia entre los años 2016 y 2019}

A continuación, en la figura 7 se muestra una comparación en cuanto al manejo de contenidos y habilidades pedagógicas del profesor durante el curso de Pirometalurgia dictado en la UTFSM en los años 2016 y 2019. Las preguntas fueron evaluadas cuantitativamente en una escala de 0 a 4 (una calificación de 3 es considerada muy baja).

Figura 7. Evaluación docente en cuanto al manejo de contenidos y habilidades pedagógicas (años 2016 y 2019)

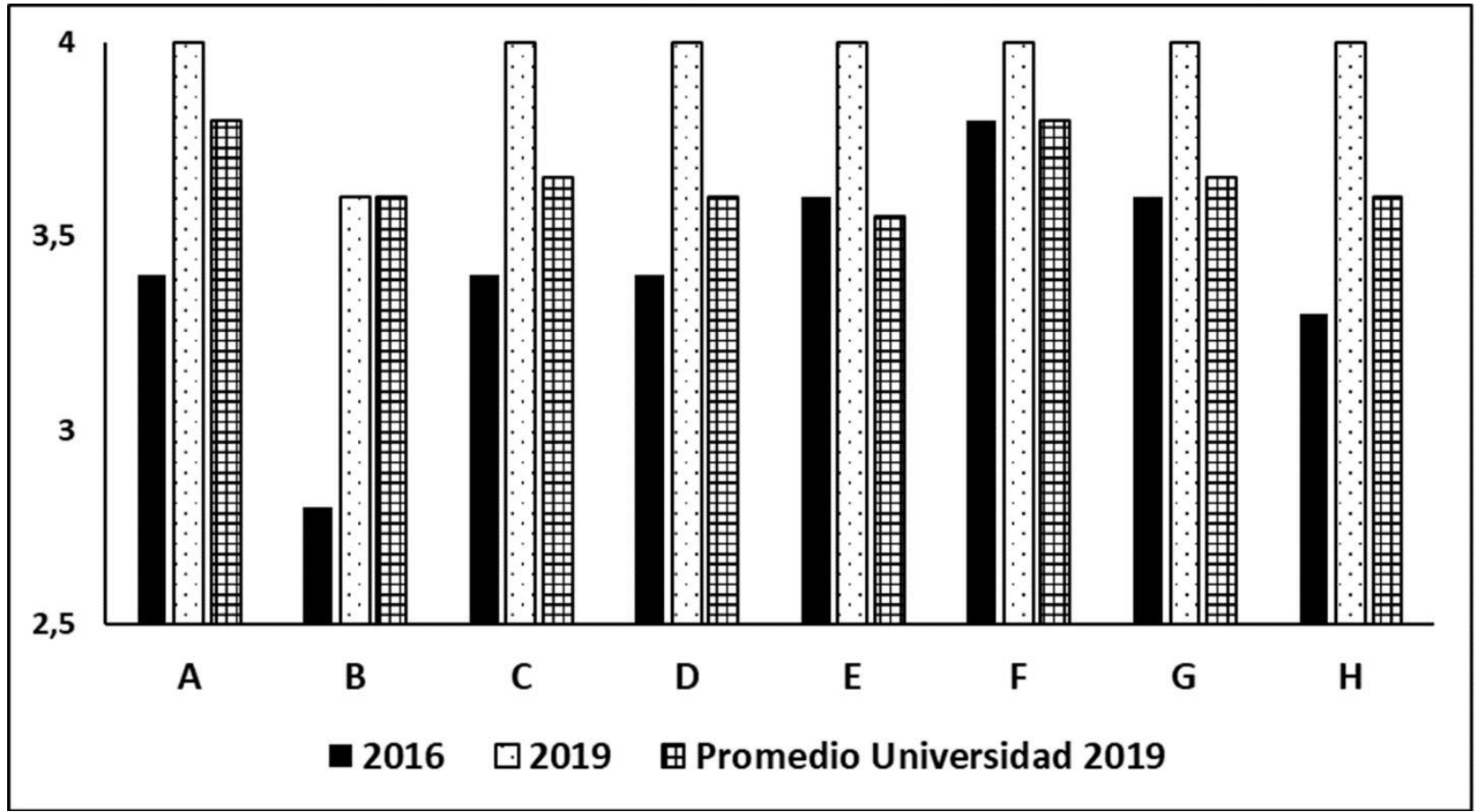

Fuente: Elaboración propia

En la tabla 3 se muestran las relacionadas sobre el manejo de contenidos y habilidades pedagógicas. Vale aclarar que el mismo profesor tuvo a cargo los cursos de 2015, 2016 y 2019. En el estudio se consideró que la diferencia en las calificaciones obtenidas entre los años 2016 y 2019 corresponde principalmente al método de enseñanza-aprendizaje utilizado. 


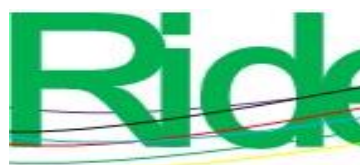

Revista Iberoamericana para la Investigación y el Desarrollo Educativo

ISSN $2007-7467$

Tabla 3. Preguntas de manejo de contenidos y habilidades pedagógicas (años 2016 y 2019)

\begin{tabular}{|l|l|}
\hline Pregunta & Contenido \\
\hline A & Mostró dominio de los temas del curso. \\
\hline B & Transmitió los contenidos del curso de forma clara y compresible. \\
\hline C & Creó un ambiente favorable del curso en el aprendizaje. \\
\hline D & Estimuló la participación activa de los estudiantes en las clases. \\
\hline E & Promovió el diálogo entre los estudiantes. \\
\hline F & Fue respetuoso con los estudiantes. \\
\hline G & Estuvo abierto a recibir críticas y sugerencias de los estudiantes. \\
\hline H & Mostró compromiso con el proceso de aprendizaje. \\
\hline I & ¿Cuánto aprendiste en el curso? \\
\hline
\end{tabular}

Fuente: Elaboración propia

En la figura 8 se observa una mejor calificación de los estudiantes sobre la labor docente en el año lectivo 2019 en comparación con el año 2016. Esta puede ser atribuida a la implementación de las intervenciones educativas usadas y comentadas anteriormente en el curso. Además, se puede apreciar que la evaluación docente del profesor en el año 2019 supera el valor promedio obtenido para el profesorado de la UTFSM en este año.

Figura 8. Resultados en torno al criterio ¿Cuánto aprendiste en el curso?

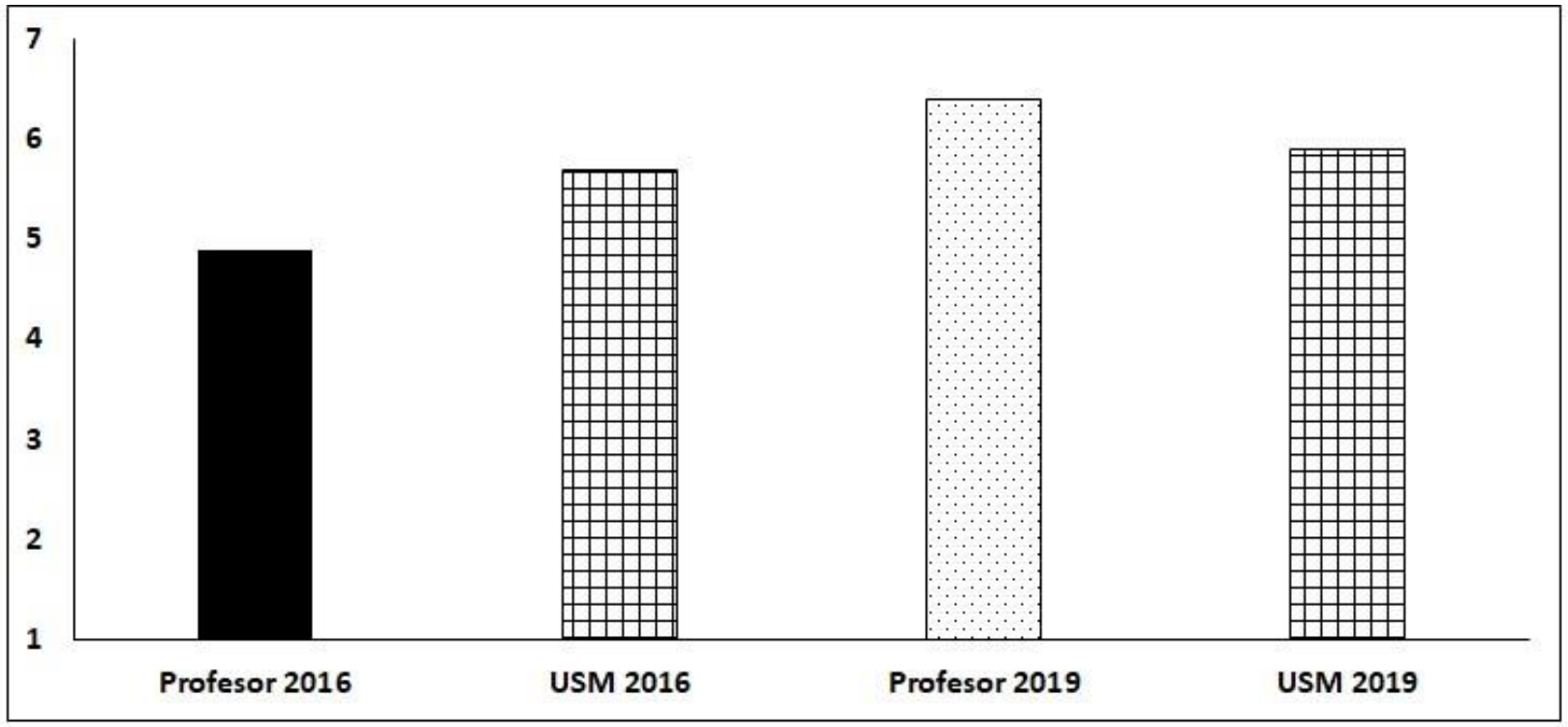

Fuente: Elaboración propia 


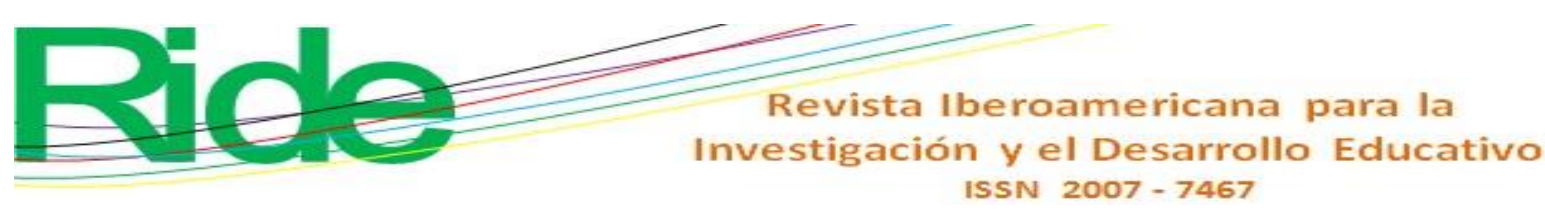

La figura 9 muestra la evaluación docente global constituida por tres preguntas: manejo de contenidos y habilidades pedagógicas, relación profesor-estudiante y aspectos formales (años 2016 y 2019) y la evaluación docente promedio de los cursos de la UTFSM en estos años (se aclara que bajo la categoría de aspectos formales se califica al profesor en los siguientes aspectos: cumplimiento con las actividades planificada, asistencia a clases con regularidad y puntualidad, distribución correcta del tiempo del curso, utilización de criterios de evaluación claros y explícitos, evaluaciones ajustadas a las actividades desarrolladas, entrega de calificaciones en los plazos establecidos y preparación de las clases).

El curso de Pirometalurgia 2019 fue mejor evaluado que el valor promedio de los cursos de la UTFSM. Es interesante señalar que ninguna pregunta de la encuesta cuantitativa fue ponderada por debajo de 4, contrario a 2016 donde aproximadamente $15 \%$ de los estudiantes consideró deficiente (menor de 4) cada una de las preguntas.

Figura 9. Evaluación global de la encuesta docente

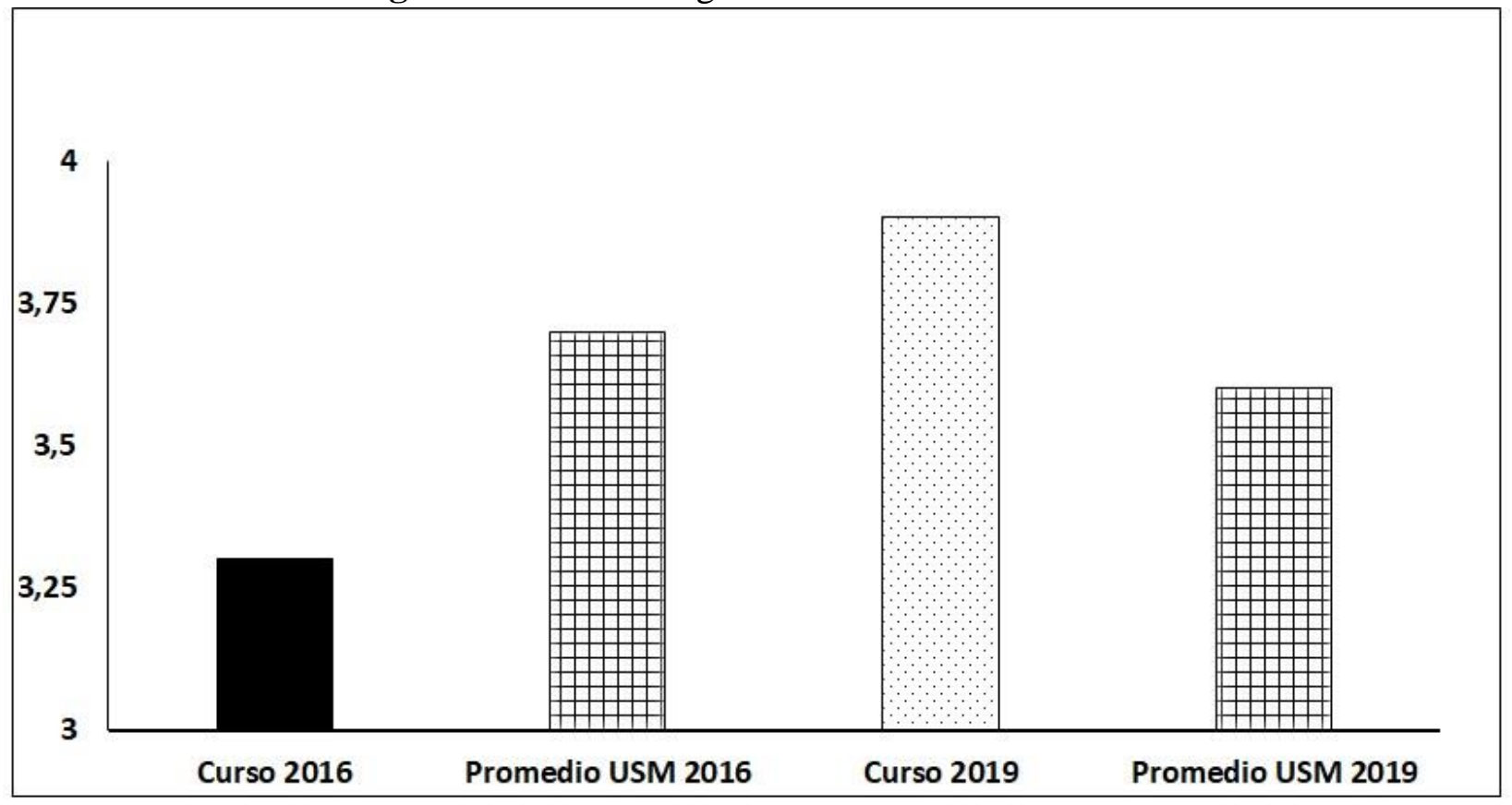

Fuente: Elaboración propia 


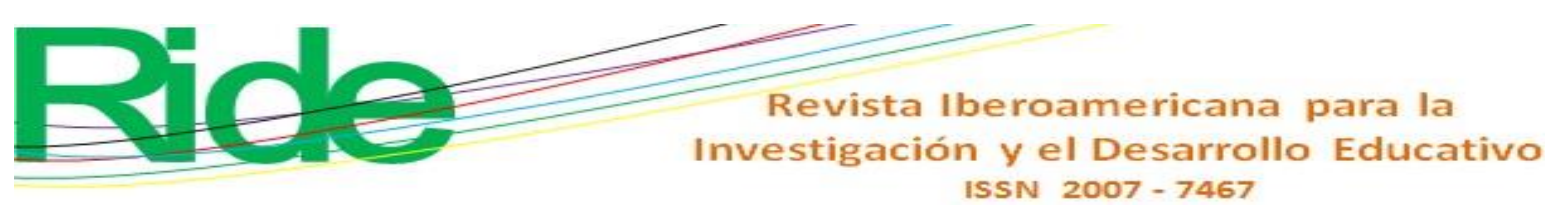

En cuanto a las limitaciones para implementar el método, se presentaron dificultades de tipo logístico tales como una inadecuada dotación de las aulas de clase. El sistema de iluminación, ventilación y aislamiento acústico no contribuyeron a mantener las mejores condiciones de trabajo. En algunas clases, las salas no disponían de mesas redondas y pizarras móviles para facilitar el trabajo grupal. Además, los ayudantes de curso, pilar básico en las discusiones grupales, carecían de capacitación y experiencia para participar en cursos de metodologías activas.

El rediseño experimental no incluyó a un grupo de control debido al pequeño número de estudiantes por año (entre 20 y 30). Esto hubiera permitido en un mismo periodo una comparación directa de las innovaciones implementadas. Por tanto, el estudio se circunscribe a comparar los resultados entre diferentes años donde el curso fue asignado al mismo profesor.

Para posteriores estudios, sin embargo, se debería considerar una evaluación sumativa estándar, pues esto permitiría hacer una comparación cuantitativa de los logros en los objetivos de aprendizaje entre los cursos impartidos en los años 2015 y 2016 (clase magistral) y 2019 (clase de metodologías activas).

El trabajo, por otra parte, no elaboró un perfil psicológico de los estudiantes al inicio del curso. Los resultados podrían contribuir a detectar estudiantes con dificultades para aceptar trabajos grupales y definir estrategias de enseñanza-aprendizaje acorde a esto. Dichos análisis serán empleados en estudios posteriores para profundizar en las mejoras de la implementación de las metodologías.

En la actualidad, el uso de metodologías activas declarado por la UTFSM como modelo educativo ya se ha implementado en algunos cursos de física. Ha sido la resistencia tanto de los docentes como de los estudiantes a cambiar los métodos tradicionales de enseñanza lo que no ha permitido su extensivo uso. Se considera que esta experiencia en el curso de Pirometalurgia 2019 puede expandirse con buen éxito a los cursos de hidrometalurgia y procesamiento de minerales que hacen parte de la malla curricular del ingeniero civil metalúrgico de la UTFSM. 


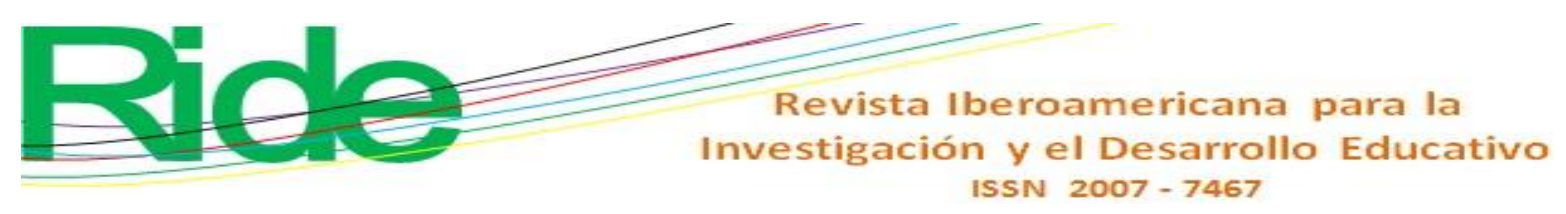

\section{Conclusiones}

La innovación en la metodología de enseñanza de la asignatura Pirometalurgia, a través de metodologías activas, se describe como un proceso que comienza con la identificación de necesidades para el aprendizaje de conceptos y enseñanzas centrales del ramo. Este derivó en una planificación didáctica y en un diseño instruccional que orientan un tránsito paradigmático, no solo metodológico y técnico.

La innovación contempló las opiniones de los estudiantes en la identificación de necesidades para el aprendizaje, así como para la planificación didáctica y el diseño de las actividades. Lo anterior fue sustentado en un diseño metodológico cualitativo descriptivo exploratorio, que otorgó una aproximación sistemática y científica a lo referido por los estudiantes, de tal forma que permitió la elaboración de categorías que orientaron el diseño mencionado.

La innovación asociada a la secuencia didáctica en aula y las actividades que implican resultó posible de implementarse en el tiempo dispuesto para la clase y según planificación. A través del contenido del discurso, los estudiantes ponderaron y sometieron a crítica las actividades grupales, las explicaciones del profesor, la discusión plenaria, las actividades individuales de simulación y la revisión de material.

La innovación metodológica desarrollada se ha fundamentado en el modelo educativo de la UTFSM que reconoce al constructivismo como eje en la comprensión de los métodos de enseñanza y el aprendizaje. Nuestra innovación acercó los procesos de diseño y evaluación metodológica a los estudiantes, siendo consonante con el modelo educativo y la racionalidad que de él se desprende.

La innovación implementada da cuenta que el aprendizaje ocurre en un medio social, en interacción con pares y en una relación con el profesor. Este último es determinante en el aprendizaje no solo por el dominio del conocimiento formal de la disciplina, sino por el buen trato,

consideración y reconocimiento hacia aquel que busca e intenta construir el saber, vale decir, sus estudiantes. De acuerdo con la experiencia obtenida en el desarrollo de esta investigación, acompañada de una cuidadosa planificación del curso, las metodologías activas de enseñanzaaprendizaje requieren para su implementación relaciones de poder planas profesor-estudiante y, por tanto, habilidades pedagógicas diferentes a las empleadas en las clases magistrales. 


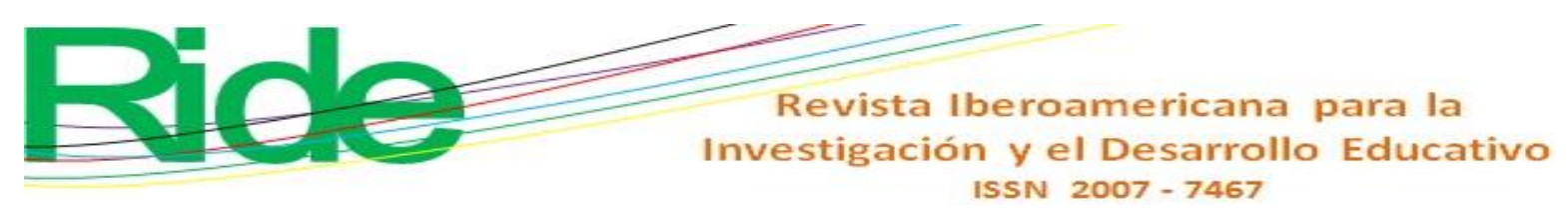

\section{Referencias}

Ausubel, D. P., Novak, J. D. y Hanesian, H. (1983). Psicología educativa: un punto de vista cognoscitivo. México: Trillas.

Bardin, L. (2002). Análisis de contenido. Madrid: Akal.

Cowan, J. and Cherry, D. (2012). The Learner's Role in Assessing Higher Level Abilities, Practitioner Research in Higher Education, 6(1), 12-22. Retrieved from https://pdfs.semanticscholar.org/af09/cbc905163f93fcd3c10ec6a571d7628859f2.pdf.

Espinoza, O. X., Silva, C. R. y López, D. E. (2017). Metodología de enseñanza activa para mejorar el rendimiento académico y el desarrollo de competencias en termodinámica a nivel de pregrado en carreras de ingeniería.

http://www.uploads/2017/12/SOCHEDI2017_paper_30.pdf.

Hewson, P. and Beckett, M. (1984). The role of conceptual conflict in conceptual change and the design of science instruction. Instructional Science, 13(1), 1-13. Retrieved from https://linkspringer-com.ezproxy.library.uq.edu.au/article/10.1007/BF00051837.

Irzik, G. (2000). Back to Basics: A Philosophical Critique of Constructivism. Science and Education, 9, 621-639. Retrieved from https://link-springercom.ezproxy.library.uq.edu.au/article/10.1023/A\%3A1010393620547.

Lewis, E. and Linn, M. (2003). Heat Energy and Temperature Concepts of Adolescents, Adults, and Experts: Implications for Curricular Improvements. Journal of Research in Science Teaching, 31(6), 657-677. Retrieved from https://onlinelibrarywileycom.ezproxy.library.uq.edu.au/doi/abs/10.1002/tea.3660310607.

Martini, S. and Hartzell, C. (2015). Integrating Computational Chemistry into a Course in Classical Thermodynamics. Journal Chemical Education, 92, 1201-1203. Retrieved from https://pubs-acs-org.ezproxy.library.uq.edu.au/doi/abs/10.1021/ed500924u.

Mazur, E. (2009). Farewell, lecture. Science, 323, 50-51. Retrieved from http://njms.rutgers.edu/education/office_education/faculty/prot/documents/FarewellLectur e-Mazur.pdf.

Mills, J. and Treagust, D. (2003). Engineering education - is problem based or project- based learning the answer. Australasian J. of Engng. Educ., 3(2), 2-16. Retrieved from http://www.aaee.com.au/journal/2003/mills_treagust03.pdf.

Salgado, A. (2007). Investigación cualitativa: diseños, evaluación del rigor metodológico y retos.
Liberabit,
13,
71-78.
Recuperado
de 


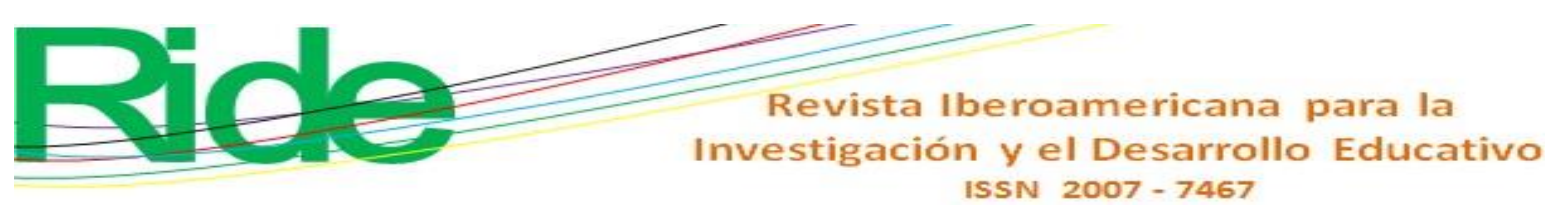

http://revistaliberabit.com/en/revistas/RLI_13_1_quality-investigation-designs-evaluationof-the-methodological-strictness-and-challenges.pdf.

Touraine A. (2012). Crítica a la modernidad. México: Fondo Cultura Económica de España.

Universidad Técnica Federico Santa María [UTFSM] (2013). Perfil de egreso ingeniero civil metalúrgico. Recuperado de http://metalurgia.usm.cl/met/perfil-de-egreso/.

Universidad Técnica Federico Santa María [UTFSM] (2015). Modelo educativo institucional. Recuperado de http://dea.usm.cl/wp-content/uploads/2018/04/modelo_ok.pdf.

Universidad Técnica Federico Santa María [UTFSM] (2017). Plan de estudios de la carrera de Ingeniería Civil Metalurgia y Materiales. Recuperado de http://www.usm.cl/admision/carreras/casa-central/ingenieria-civil-metalurgica/.

Valdivia, C. G. (2008). El internista en la práctica clínica habitual. Problemas y soluciones. Santiago de Chile, Chile: Departamento de Salud Pública. Pontificia Universidad Católica de Chile. Recuperado de https://es.scribd.com/document/431232417/El-internista-en-lapractica-clinica-habitual-problemas-y-soluciones-el-enfoque-descriptivo-pdf 


\begin{tabular}{|c|c|}
\hline Rol de Contribución & Autor (es) \\
\hline Conceptualización & $\begin{array}{l}\text { Héctor Henao (igual), Alejandra Chávez (igual), Juan Ibáñez } \\
\text { (apoya) }\end{array}$ \\
\hline Metodología & Hector Henao (Igual), Alejandra Chávez (igual) \\
\hline Software & Christian García \\
\hline Validación & Christian García (principal), Héctor Henao (apoya) \\
\hline Análisis Formal & Christian García (principal) y Héctor Henao (apoya) \\
\hline Investigación & $\begin{array}{l}\text { Héctor Henao (principal), Juan Ibáñez (apoya), Claudio Pizarro } \\
\text { (apoya), Alejandra Chávez (apoya) }\end{array}$ \\
\hline Recursos & Héctor Henao (igual), Patricio Ibáñez (igual) \\
\hline Curación de datos & Héctor Henao (principal), Alejandra Chávez (apoya) \\
\hline $\begin{array}{l}\text { Escritura - Preparación del } \\
\text { borrador original }\end{array}$ & $\begin{array}{l}\text { Hector Henao (igual), Alejandra Chávez (igual), Christian } \\
\text { García (igual) }\end{array}$ \\
\hline $\begin{array}{l}\text { Escritura - Revisión y } \\
\text { edición }\end{array}$ & $\begin{array}{l}\text { Héctor Henao (principal), Alejandra Chávez (apoya), Christian } \\
\text { García (apoya), Claudio, Pizarro (apoya), Adriana Ruiz (apoya) }\end{array}$ \\
\hline Visualización & Claudio Pizarro (igual), Juan Ibáñez (igual) \\
\hline Supervisión & Héctor Henao (principal), Juan Ibáñez (apoya) \\
\hline $\begin{array}{l}\text { Administración } \\
\text { Proyectos }\end{array}$ & Héctor Henao (principal), Juan Ibáñez (apoya) \\
\hline Adquisición de fondos & $\begin{array}{l}\text { Héctor Henao (principal), Juan Ibáñez (apoya), Alejandra } \\
\text { Chávez (apoya) }\end{array}$ \\
\hline
\end{tabular}

